\title{
Apports spontanés en acides gras oméga 3 chez des diabétiques de type 2 tunisiens
}

\author{
Henda Jamoussi ${ }^{\star}$, Salma Chaabouni, Amel Gammoudi, Faten Mahjoub, Kamilia Ounaissa, Olfa Berriche, \\ Chiraz Amrouche et Samira Blouza
}

Service de nutrition, diabétologie et maladies métaboliques, Institut national de nutrition et de technologie alimentaire de Tunis, Bab Saadoun, 1007 Tunis, Tunisie

Reçu le 13 janvier 2014 - Accepté le 12 mai 2014

\begin{abstract}
Résumé - Il est admis à l'unanimité que les acides gras oméga 3 ont de multiples bénéfices pour la santé et plus précisément des effets protecteurs contre les maladies cardiovasculaires et neurodégénératives. L'objectif de ce travail était d'estimer les apports spontanés en acides alphalinolénique (ALA), docosahexaénoïque (DHA), éicosapentaénoïque (EPA) et linoléique (LA). Cette étude d'observation a été réalisée auprès de 42 diabétiques de type 2 (21 hommes et 21 femmes) âgés de 30 à 75 ans. L'estimation des apports en acides gras a été réalisée en utilisant la méthode d'enregistrement sur sept jours. Les résultats ont montré que l'apport moyen en ALA était de 0,99 $\pm 0,40 \mathrm{~g} / \mathrm{j}$ soit $0,42 \pm 0,13 \%$ de l'apport énergétique total (AET). Aucun de nos patients n'avait un apport suffisant en ALA. Les apports quotidiens moyens en DHA et en EPA étaient respectivement de $90,0 \pm 85 \mathrm{mg} / \mathrm{j}$ et de $83,5 \pm 80 \mathrm{mg} / \mathrm{j}$. Quant à la contribution des aliments aux apports en EPA et en DHA, les poissons et les fruits de mer représentaient la part la plus importante $(57 \%)$ de ces deux acides gras. Nos résultats appellent à renforcer l'éducation nutritionnelle des diabétiques afin d'augmenter les apports en acides gras oméga 3 et d'assurer un rapport LA/ALA optimal qui contribuerait à la prévention des maladies cardiovasculaires.
\end{abstract}

Mots clés : Acides gras polyinsaturés / rapport oméga 6/oméga 3 / diabète type 2 / risque cardiovasculaire

\begin{abstract}
Spontaneous intakes of omega 3 fatty acids in tunisian type 2 diabetics. It is unanimously agreed that the omega 3 fatty acids have many health benefits and specifically, protective effects against cardiovascular and neurodegenerative diseases. The objective of this work was to estimate the spontaneous intake of alphalinolenic, docosahexaenoique (DHA), eicosapentaenoique (EPA) and linoleic acids. This observational study was conducted among 42 type 2 diabetics ( 21 men and 21 women) aged from 30 to 75 years. To estimate spontaneous intakes of polyinsaturated fatty acids, we used the seven days recording method. Our results showed the average daily intake of ALA was $0.99 \pm 0.40 \mathrm{~g} / \mathrm{d}$ or $0.42 \pm 0.13 \%$ of total energy intake. None of our patients had an adequate intake of ALA. Average daily intakes of DHA and EPA, were respectively $90.0 \pm 85 \mathrm{mg} / \mathrm{d}$ and $83.5 \pm 80 \mathrm{mg} / \mathrm{d}$. As for the contribution of food to intake of EPA and DHA, fish and seafood accounted for the largest share (57\%) of these fatty acids. Our results justify to strengthen nutritional education of diabetic subjects to increase the consumption of foods rich in omega 3 fatty acids and to provide an adequate report LA/ALA to multiple benefits against protection of cardiovascular diseases.
\end{abstract}

Keywords: Polyunsaturated fatty acids / omega 6/omega 3 ratio / type 2 diabetes / cardiovascular risk

\section{Introduction}

Les acides gras polyinsaturés (AGPI) sont les principaux constituants des membranes cellulaires et des sources d'énergie. Ils sont également nécessaires dans la synthèse de nombreuses substances impliquées dans des processus biologiques (la coagulation, l'inflammation, l'immunité...) (Legrand, 2007).

L'acide alpha-linolénique (ALA) et l'acide linoléique (LA), chefs de file des AGPI oméga 3 et oméga 6 respective-

\footnotetext{
^ Correspondance : hendajamoussi@gmail.com
}

ment, sont des acides gras essentiels, car l'organisme est incapable de les synthétiser. Les acides éicosapentaénoïque (EPA) et docosahexaénoïque (DHA) d'une part et l'acide arachidonique d'autre part, sont des dérivés supérieurs provenant respectivement de l'ALA et du LA (Lecerf, 2004). Dans l'organisme, la biotransformation de l'ALA en EPA puis en DHA est faible notamment en cas de diabète sucré (Raccah, 1997).

Les études épidémiologiques d'observation et d'intervention s'accordent sur les bénéfices multiples des acides gras oméga 3, et plus précisément, sur leurs effets protecteurs contre de nombreuses maladies (cardiovasculaires, 
dégénérescence maculaire, neuropathie diabétique, maladie d'Alzheimer...) (Nettleton, 2005). Dans sa revue de la littérature, Nettleton a rapporté les résultats d'études cliniques contrôlées qui ont montré que la consommation des acides gras oméga 3 à longues chaînes, à raison de 1 à $2 \mathrm{~g} / \mathrm{j}$, a des effets cardioprotecteurs chez les diabétiques de type 2 et pourrait réduire le risque de conversion de l'intolérance au glucose vers un diabète patent (Nettleton, 2005). Toutefois, les données récentes d'une grande étude réalisée par le groupe d'experts en nutrition et maladies chroniques (NutriCoDE) dans 187 pays ont montré un faible niveau de consommation des acides gras oméga 3 dans la plupart des pays. En effet, l'apport en ALA est inférieur à 0,5\% de l'apport énergétique total dans 61 pays. Quant aux acides gras oméga 3 d'origine marine, leur apport est inférieur à $100 \mathrm{mg} /$ jour dans 100 pays (l'Afrique sub-saharienne, l'Afrique du Nord, les régions asiatiques et le Moyen-Orient) représentant $80 \%$ de la population adulte mondiale (Micha, 2014).

En Tunisie, jusqu'à présent aucune étude ne s'est intéressée à l'estimation des apports en AGPI oméga 3 et 6 chez des diabétiques de type 2 . Ceci nous a mené à réaliser cette étude au sein d'une population de diabétiques de type 2 qui sont à haut risque cardiovasculaire. Les objectifs de ce travail étaient d'estimer les apports spontanés en acides alphalinolénique (ALA), docosahexaénoïque (DHA), éicosapentaénoïque (EPA) et linoléique (LA) et d'étudier les facteurs influençant les apports en ces différents acides gras.

\section{Patients et méthodes}

Cette étude transversale descriptive a concerné 42 diabétiques de type 2 suivis aux consultations externes de diabétologie de l'Institut national de nutrition et recrutés sur une période de deux mois (février-mars 2013).

Pour l'évaluation des apports nutritionnels spontanés, nous avons opté pour la méthode du semainier. L'enregistrement de la consommation alimentaire de sept jours consécutifs a été réalisé par les patients eux mêmes puis vérifié au cours d'un entretien avec la diététicienne du service. Cette enquête nous a permis de déterminer le type et la quantité d'aliments consommés et la fréquence de leur consommation durant toute la semaine. Elle nous a permis également de préciser le mode de cuisson (friture, grillade... ) et la nature de l'huile utilisée pour la cuisson et l'assaisonnement. Nous avons utilisé les mesures usuelles (bol, tasse, verre, cuillère à soupe, cuillère à café...) pour estimer les portions d'aliments et pour la quantification des apports.

Nous avons utilisé un logiciel spécifique «Bilnut» version 1991 pour saisir les données de l'enquête et établir le bilan nutritionnel. Le bilan nutritionnel comprend : l'apport calorique total spontané, la répartition des nutriments énergétiques (protides, glucides et lipides) dans la journée, la répartition de la ration lipidique en acides gras saturés, monoinsaturés et polyinsaturés et l'apport des autres nutriments (cholestérol, sels minéraux, vitamines et fibres alimentaires). Pour l'estimation des apports spontanés des acides gras polyinsaturés, et en raison de l'absence, dans la table de composition tunisienne, des teneurs des aliments en ces acides gras, nous avons utilisé les données du Centre français d'information sur la qualité des aliments (CIQUAL, 2012). Par ailleurs, les données sur les teneurs en AGPI essentiels et la variation de la composition de certains poissons blancs selon le mode de cuisson sont issues des publications de recherches effectuées à la faculté de médecine de Monastir (Mnari Bhouri, 2009). Concernant les aliments enrichis en AGPI oméga 3 disponibles sur le marché tunisien et qui sont consommés par nos diabétiques, leur composition en acides gras a été fournie par l'étiquetage présent sur l'emballage.

Tous nos patients ont bénéficié d'un interrogatoire minutieux pour préciser l'ancienneté et l'existence d'éventuelles complications du diabète. Le niveau socioéconomique a été estimé en se basant principalement sur le niveau de scolarité, la profession et les revenus des parents. Le poids et la taille ont été mesurés pour calculer l'indice de masse corporelle $\left(\mathrm{IMC}=\right.$ poids $(\mathrm{kg}) /$ taille $\left.(\mathrm{m})^{2}\right)$. Le surpoids a été défini par un IMC $\geqslant 25 \mathrm{~kg} / \mathrm{m}^{2}$ et l'obésité par un IMC $\geqslant 30 \mathrm{~kg} / \mathrm{m}^{2}$.

Les analyses statistiques ont été réalisées à l'aide du logiciel «EPI INFO » version 2000 en utilisant les tests de Mann et Whitney pour la comparaison de deux moyennes et celui de Kruskal et Wallis pour la comparaison de plusieurs moyennes. Ces tests non paramétriques ont été utilisés en raison de la petite taille des échantillons $(n<30)$. Une différence est considérée comme statistiquement significative si la valeur de $p$ est inférieure à 0,05 .

\section{Résultats}

L'âge moyen de nos patients était de 54,8 \pm 8 ans. Les trois quarts $(76 \%)$ d'entre eux avaient un surpoids dont $44 \%$ étaient obèses. Les autres caractéristiques de la population étudiée sont récapitulées dans le Tableau 1.

L'analyse de l'alimentation spontanée de la population étudiée a montré un apport énergétique total moyen de $2031 \pm$ $227 \mathrm{kcal} / \mathrm{j}$. La ration lipidique moyenne était de 34,8 $\pm 5,8 \%$ de l'apport énergétique total (AET). Elle était répartie en $9,8 \pm 3 \%$ d'acides gras saturés, 19,5 $\pm 5 \%$ d'acides gras monoinsaturés et $8,8 \pm 4 \%$ d'acides gras polyinsaturés (Tab. 2). Aucune différence significative n'a été observée entre les apports nutritionnels des hommes et ceux des femmes.

L'apport quotidien moyen en acide alphalinolénique (ALA) était de $0,99 \pm 0,4 \mathrm{~g} / \mathrm{j}$ soit $0,42 \pm 0,13 \%$ de l'apport énergétique total. L'apport journalier moyen en DHA était de $90 \mathrm{mg} \pm 85 \mathrm{mg} / \mathrm{j}$. Cet apport n'est satisfaisant $(250 \mathrm{mg})$ que chez seulement 5,8\% des patients. Quant à l'apport en EPA, il était de $83,5 \pm 80 \mathrm{mg} / \mathrm{j}$. Aucun de nos patients n'avait un apport optimal en EPA. Un apport total en DHA + EPA, dépassant $500 \mathrm{mg} / \mathrm{j}$, était observé chez un seul patient (soit 2,9\%).

Quant à l'apport quotidien moyen en acide linoléique (LA), il était de $15 \pm 6 \mathrm{~g} / \mathrm{j}$ soit $6,99 \pm 2,8 \%$ de l'AET. Il dépassait les apports recommandés (4\% de l'AET) dans 67,5\% des cas et seulement $15 \%$ de nos patients avaient un apport optimal.

Le rapport LA/ALA moyen était de $17 \pm 8$ avec des extrêmes allant de 6,5 à 38 . Aucun de nos patients ne présentait un rapport optimal $(<5)$. Ce rapport dépassait $10 \mathrm{chez}$ les trois quarts de nos patients $(77,5 \%)$. 
Tableau 1. Caractéristiques générales de la population étudiée.

\begin{tabular}{cccc}
\hline Caractéristiques & $\begin{array}{c}\text { Hommes } \\
(n=21)\end{array}$ & $\begin{array}{c}\text { Femmes } \\
(n=21)\end{array}$ & $\begin{array}{c}\text { Total } \\
(n=42)\end{array}$ \\
\hline Âge moyen (ans) & $55,0 \pm 6$ & $54,0 \pm 10$ & $54,8 \pm 8$ \\
Niveau socioéconomique $(\%)$ & & & \\
Moyen \% $(n)$ & $62(13)$ & $57(12)$ & $60(25)$ \\
Élevé \% $(n)$ & $38(8)$ & $43(9)$ & $40(17)$ \\
Origine côtière \% $(n)$ & $48,0(10)$ & $57,0(10)$ & $52,5(22)$ \\
IMC moyen $\left(\mathrm{kg} / \mathrm{m}^{2}\right)$ & $27 \pm 4,0$ & $29 \pm 3,6$ & $28 \pm 4,0$ \\
Durée d'évolution & $8,5 \pm 5,0$ & $10,5 \pm 4,8$ & $10,0 \pm 5,0$ \\
moyenne du diabète $($ ans $)$ & & & \\
Taux moyen d'HbA1c $(\%)$ & $8,5 \pm 1,3$ & $7,8 \pm 0,9$ & $8,2 \pm 1,2$ \\
Hypertension artérielle \% $(n)$ & $57(12)$ & $62(13)$ & $60(25)$ \\
Dyslipidémie \% $(n)$ & $71(15)$ & $67(14)$ & $69(29)$ \\
Maladie coronaire \% $(n)$ & $19,0(4)$ & $9,5(2)$ & $15,0(6)$ \\
\hline
\end{tabular}

$\mathrm{P}=\mathrm{NS}$ : Absence de différence significative entre les hommes et les femmes pour toutes les variables.

Tableau 2. Apports énergétiques et en macronutriments selon le sexe.

\begin{tabular}{ccc}
\hline Nutriment & $\begin{array}{c}\text { Hommes }(n=21) \\
\text { Moyenne }(\mathrm{ET})\end{array}$ & $\begin{array}{c}\text { Femmes }(n=21) \\
\text { Moyenne }(\mathrm{ET})\end{array}$ \\
\hline Apport énergétique total (AET)(Kcal/j) & $2012(228)$ & $2050(230)$ \\
Glucides totaux (\% AET) & $50,00(5,0)$ & $48,00(6,0)$ \\
Lipides totaux (\% AET) & $35,70(5,0)$ & $39,85(6,8)$ \\
Protides totaux (\% AET) & $13,30(1,5)$ & $12,60(1,8)$ \\
Acides gras saturés (\%) & $9,80(4,0)$ & $9,90(2,0)$ \\
Acides gras monoinsaturés (\%) & $17,20(5,3)$ & $22,00(4,8)$ \\
Acides gras polyinsaturés $(\%)$ & $9,50(3,6)$ & $8,00(5,6)$ \\
\hline
\end{tabular}

ET : écart-type. $\mathrm{P}=\mathrm{NS}$ : Absence de différence significative entre les hommes et les femmes pour tous les apports nutritionnels.

Tableau 3. Facteurs influençant les apports en acides gras polyinsaturés essentiels et leur rapport.

\begin{tabular}{cccccc}
\hline Facteurs & $\begin{array}{c}\text { ALA (\%AET) } \\
\text { Moyenne (ET) }\end{array}$ & $\begin{array}{c}\text { LA (\% AET) } \\
\text { Moyenne (ET) }\end{array}$ & $\begin{array}{c}\text { DHA(mg/j) } \\
\text { Moyenne (ET) }\end{array}$ & $\begin{array}{c}\text { EPA (mg/j) } \\
\text { Moyenne (ET) }\end{array}$ & $\begin{array}{c}\text { LA/ALA } \\
\text { Moyenne (ET) }\end{array}$ \\
\hline Sexe & & & & \\
Hommes $(n=21)$ & $0,45(0,14)$ & $8,20(2,86)$ & $94,0(72,0)$ & $95,0(86,0)$ & $18,8(9,1)$ \\
Femmes $(n=21)$ & $0,39(0,11)$ & $5,80(2,50)$ & $84,0(30,0)$ & $70,0(87,0)$ & $15,6(8,5)$ \\
$P$ & $\mathrm{NS}$ & $\mathrm{NS}$ & $\mathrm{NS}$ & $\mathrm{NS}$ & $\mathrm{NS}$ \\
\hline Tranches d'âge & & & & \\
$<45$ ans $(n=11)$ & $0,28(0,08)$ & $5,50(2,30)$ & $90,7(106,0)$ & $92,6(97,5)$ & $20,2(11,8)$ \\
$45-65$ ans $(n=19$ & $0,42(0,10)$ & $7,30(3,00)$ & $75,5(73,1)$ & $73,6(81,5)$ & $17,3(9,6)$ \\
$>65$ ans $(n=12)$ & $0,48(0,11)$ & $5,38(0,70)$ & $198,3(84,8)$ & $136,0(108,0)$ & $11,0(3,6)$ \\
$P$ & $\mathrm{NS}$ & $\mathrm{NS}$ & $\mathrm{NS}$ & $\mathrm{NS}$ & $\mathrm{NS}$ \\
\hline Niveau socioéconomique & & & & \\
Moyen $(n=25)$ & $0,38(0,10)$ & $7,90(2,50)$ & $83,5(78,0)$ & $78,4(86,0)$ & $20,3(8,7)$ \\
Bon $(n=17)$ & $0,48(0,15)$ & $6,00(3,00)$ & $98,6(100,0)$ & $90,6(89,0)$ & $12,5(6,8)$ \\
$p$ & 0,02 & $\mathrm{NS}$ & $\mathrm{NS}$ & $\mathrm{NS}$ & 0,001 \\
\hline
\end{tabular}

L'apport moyen en ALA augmentait avec l'âge, mais sans différence statistiquement significative. Les diabétiques âgés de plus de 65 ans consommaient plus d'acides gras oméga 3 et moins d'acides gras oméga 6 , et par conséquent leurs rapports étaient les plus bas par rapport aux autres patients moins âgés (Tab. 3).

Nous avons constaté que l'apport en ALA était plus élevé chez les diabétiques qui appartiennent à une classe sociale favorisée $(0,48 \pm 0,15 \%)$ que chez ceux qui ont un niveau socioéconomique moyen $(0,38 \pm 0,1 \%)$, la différence était statistiquement significative $(p=0,02)$. Quant au rapport LA/ALA, il était plus élevé chez les diabétiques qui appartenaient à une classe sociale moyenne $(20,3 \pm 8,7)$ que chez ceux qui avaient un niveau socioéconomique élevé $(12,5 \pm 6,8)$, la différence était statistiquement significative ( $p=0,001)$ (Tab. 3).

Quant aux acides gras DHA et EPA, leurs apports n'étaient influencés ni par le sexe, ni par l'âge et ni par le niveau socioéconomique. Ces apports n'étaient pas également influencés par l'origine géographique des patients.

Enfin, nous avons constaté que les diabétiques coronariens consommaient moins d'acide alpha linoléique $(0,30 \pm 0,07 \%$ de l'AET) que les patients non coronariens $(0,44 \pm 0,13 \%$ de 


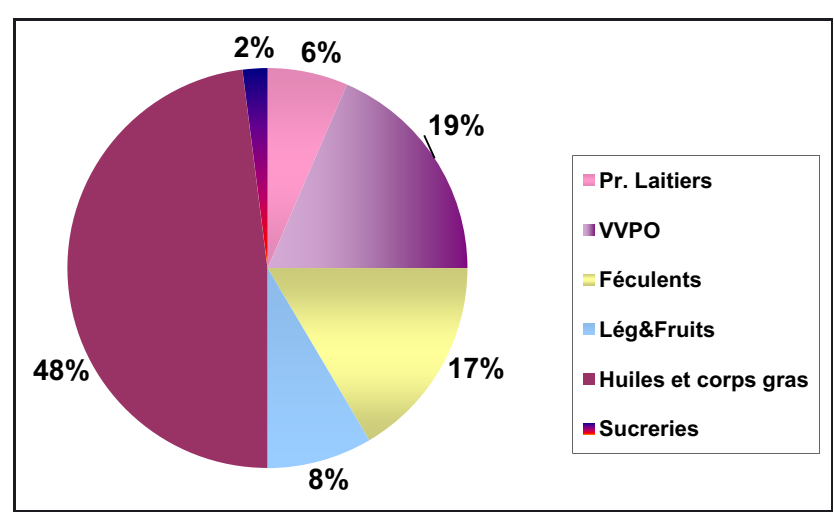

Fig. 1. Contribution des aliments aux apports en acide alphalinolénique.

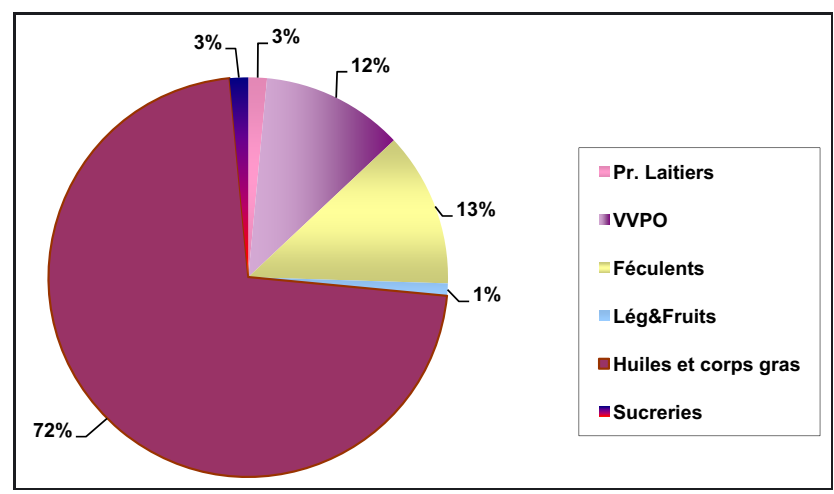

Fig. 2. Contribution des aliments aux apports en acide linoléique.

l'AET). La différence était statistiquement significative ( $p=$ 0,01). Le rapport LA/ALA était par conséquent, significativement plus élevé chez les diabétiques coronariens $(27 \pm 12)$ que chez les non coronariens $(15 \pm 7 ; p=0,005)$.

L'étude de la nature des aliments contribuant aux apports des précurseurs des AGPI a montré qu'ils étaient essentiellement apportés par les aliments d'origine végétale, avec des contributions respectives de $75 \%$ pour l'ALA et de $87 \%$ pour le LA. Les huiles et les corps gras (margarine, oléagineux...) contribuaient à la moitié de l'apport en ALA (48\%) et aux trois quarts de l'apport en LA (72\%). La part des viandes, des volailles, des poissons et des œufs (VVPO), plutôt riches en acides gras polyinsaturés à longue chaîne, ne représentaient que $18 \%$ de l'apport total en ALA (Figs. 1 et 2).

Les poissons et les fruits de mer contribuaient par la part la plus importante (57\%) aux apports en EPA et en DHA. La part des volailles est de $22 \%$. Quant aux viandes rouges et les œufs, leurs parts respectives étaient de 8 et $11 \%$ (Fig. 3).

\section{Discussion}

Les AGPI oméga 3, en raison de leurs multiples bienfaits sur la santé, notamment la protection cardiovasculaire, ont fait l'objet de nombreuses études d'observation qui ont permis l'estimation des apports spontanés en ces acides gras bénéfiques.

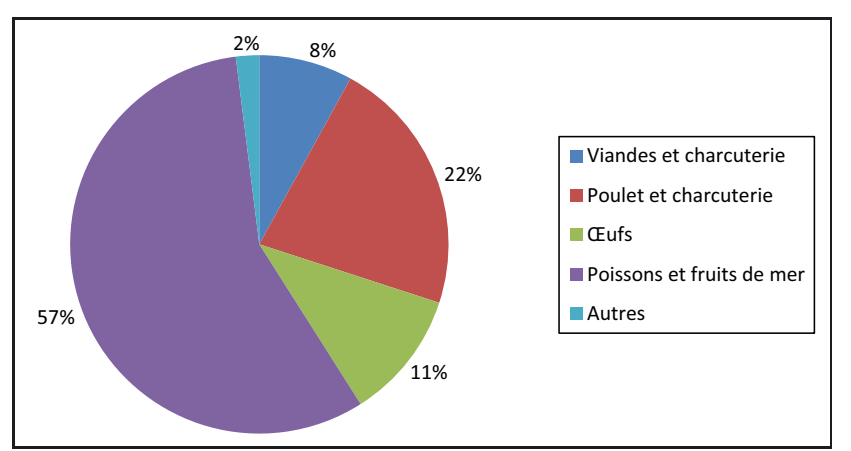

Fig. 3. Contribution des aliments aux apports en EPA et en DHA.

Notre étude a révélé, malgré une ration calorique relativement correcte sur le plan quantitatif, un déséquilibre qui concerne particulièrement les acides gras polyinsaturés. En effet, l'apport quotidien moyen en acide alphalinolénique (ALA) ne représente que $42 \%$ des apports conseillés. Aucun de nos patients ne consommait la quantité d'ALA recommandée par l'agence nationale de sécurité sanitaire de l'alimentation, de l'environnement et du travail (1\% de l'AET) (ANSES, 2011). Quant aux apports moyens en DHA et en EPA, ils sont également inférieurs aux apports conseillés pour ces deux acides gras. Le déficit d'apport en ALA s'expliquerait d'une part, par la faible consommation d'huiles végétales (huile de colza, de noix et de soja) et d'oléagineux riches en cet acide gras et d'autre part, par la faible teneur des produits d'origine animale en ALA. En outre, la faible consommation des poissons riches en oméga 3 (maquereau, thon...) explique la carence d'apport en DHA et en EPA. En revanche, la consommation importante des huiles de maïs, de tournesol et d'huile de mélange explique l'excès d'apport en acide linoléique $(15 \pm 6 \mathrm{~g} / \mathrm{j})$.

Les estimations des apports en AGPI oméga 3 et 6 , réalisées dans la population générale, ont révélé de grandes variations selon les études et les pays. Toutefois, nos résultats concordent avec ceux de la majorité des études d'observation réalisées dans le monde. L'étude SU.VI.MAX (Astorg, 2007) menée auprès de 4884 adultes français, qui ont rempli 10 relevés alimentaires de 24 h sur une période de 2,5 ans, a montré un apport moyen en ALA de 0,38 \% de l'AET. Les produits laitiers, les viandes, les volailles et les œufs sont des sources majeures d'ALA, fournissant ensemble plus de $41 \%$ de son apport. Cette étude a révélé des apports moyens en DHA $(230 \pm 140 \mathrm{mg} / \mathrm{j})$ et en EPA $(120 \pm 9 \mathrm{mg} / \mathrm{j})$ plus élevés que ceux observés dans notre étude. Ces apports sont fortement corrélés à la consommation de poissons et de fruits de mer qui contribuent par des parts respectives de $72 \%$ et $65 \%$. Les poissons gras (hareng, sardines, maquereau, saumon et thon frais) fournissent à eux seuls 33\% des apports en EPA et en DHA.

L'étude TRANSFAIR (Hulshof, 1999) a comparé les apports en acides gras d'échantillons de populations de 14 pays d'Europe. L'apport moyen en acide alphalinolénique en France est relativement bas par rapport aux autres pays européens. Chez les français, il est de $0,6 \pm 0,3 \mathrm{~g} / \mathrm{j}$ chez les hommes et de $0,5 \pm 02 \mathrm{~g} / \mathrm{j}$ chez les femmes. Il atteint $0,8 \mathrm{~g} / \mathrm{j}$ en Italie, $1,4 \pm 0,7 \mathrm{~g} / \mathrm{j}$ en Suède et $1,8 \pm 0,9 \mathrm{~g} / \mathrm{j}$ en Finlande. Des apports plus élevés en ALA ont été observés dans les pays occidentaux (États-Unis, Canada, Europe du nord, Australie); ils 
varient de 0,5 à 0,6\% de l'apport énergétique total. Au Japon, en particulier, les apports moyens en ALA se rapprochent plus des recommandations. En effet, ils étaient de 1,7-2,2 g/j ou 0,7-1\% de l'AET, en raison de l'usage répandu des huiles de colza et de soja dans ce pays. Quant aux apports en EPA et en DHA, ils étaient de 1-1,5 g/j d'EPA + DHA chez les hommes et $0,7-1,1 \mathrm{~g} / \mathrm{j}$ chez les femmes (Sugano, 2000). En Norvège et en Espagne, l'apport moyen en DHA est de l'ordre de 0,5$0,7 \mathrm{~g} / \mathrm{j}$. Ces apports élevés en acides gras oméga 3 à longue chaîne s'expliquent par la plus grande consommation de poissons dans les pays scandinaves et en Espagne que le reste de l'Europe. Les apports les plus bas en ces acides gras ont été observés aux États-Unis, au Canada, en Belgique, en Allemagne et en Australie. L'apport en DHA est de 0,1 à $0,2 \mathrm{~g} / \mathrm{j}$ dans ces pays (Ollis, 1999).

Le faible apport en ALA et la consommation assez importante d'acide linoléique expliquent l'élévation importante du rapport LA/ALA dans notre série $(17 \pm 8)$. Ce rapport est plus élevé que ceux observés dans les études TRANSFAIR (Hulshof, 1999), INCA1 (AFSSA, 2003) et SU.VI.MAX (Astorg, 2007) qui sont respectivement de 13, 12,9 et 11. Dans les pays occidentaux (États-Unis, Canada, Europe du Nord, Australie), le rapport est compris entre 6 et 10. Il est moins élevé au Japon, compris entre 4 et 8.

De nombreuses études ont suggéré que les AGPI oméga 3 ont un effet cardioprotecteur. Parmi celles-ci, une grande étude d'observation a été menée, par des chercheurs scientifiques danois et américains, sur la consommation de poissons et d'AGPI oméga 3 dans le Grand Nord chez les Esquimaux. Cette étude a démontré que ces derniers sont rarement touchés par les maladies cardiovasculaires grâce à la consommation de poissons et de mammifères marins riches en AGPI. L'analyse du sang a montré que les niveaux de cholestérol et de triglycérides sont nettement plus faibles que ceux de la population danoise dont l'alimentation est principalement basée sur les graisses animales et les laitages (ANSES, 2011).

En prévention secondaire, une étude récente menée auprès de 1014 personnes diabétiques, âgées de 60 et 80 ans et ayant eu un infarctus du myocarde, a démontré les bénéfices cardiovasculaires des acides gras oméga 3. En effet, les participants ont été répartis au hasard en quatre groupes. Dans chaque groupe, les patients devaient consommer $20 \mathrm{~g}$ de margarine par jour pendant quarante mois. Trois des margarines utilisées étaient enrichies en acides gras oméga 3 (une margarine contenait $400 \mathrm{mg}$ d'EPA/DHA, la seconde contenait $2 \mathrm{~g}$ d'ALA, la troisième contenait $400 \mathrm{mg}$ d'EPA/DHA plus $2 \mathrm{~g}$ d'ALA). Le groupe ayant reçu la margarine sans ajout d'acide gras oméga 3 a servi de groupe de contrôle. Les résultats ont montré une réduction des risques de survenue des évènements liés aux arythmies ventriculaires et de décès par infarctus du myocarde de $72 \%$ dans le groupe de diabétiques ayant reçu la margarine associant les trois acides gras oméga 3 (Kromhout, 2011).

Il ressort de l'ensemble de ces études, en dépit des imprécisions et des limites méthodologiques, que l'apport en acide alphalinolénique est insuffisant dans de nombreux pays et loin de couvrir les besoins de l'organisme et que le rapport est souvent élevé. Le caractère défavorable d'un rapport élevé est lié à une production accrue d'eicosanoides proagré- gantes (thromboxanes A2) et proinflammatoires (prostaglandines E2 et leucotriènes B4) induite par l'excès d'apport en acides gras oméga 6 . En outre, étant donné que les métabolismes des acides gras oméga 6 et oméga 3 font appel aux mêmes enzymes (désaturases et élongases), l'excès d'apport en acide linoléique empêche l'utilisation optimale des acides gras oméga 3 par l'organisme pouvant compromettre la production d'EPA et de DHA à partir de l'acide alphalinolénique. Ces acides gras à longues chaînes serviront de précurseurs pour la synthèse des prostaglandines antiagrégantes (PG E3) ou neutres (TX A3) et de leucotriènes peu inflammatoires (LT B4) (AFSSA, 2003 ; Dallongeville, 2009; Tedgui et chapman, 2003). Un rapport LA/ALA élevé est associé à des altérations de la microvascularisation, une inflammation chronique, une agrégation plaquettaire, des dysfonctionnements endothéliaux et une moindre stabilité de la plaque d'athérome qui est caractérisée par sa vulnérabilité en présence d'un diabète de type 2 avec un risque élevé de thrombose (Lecerf, 2008). En outre, chez le diabétique, il existe une diminution de l'activité de la delta 6 désaturase, première enzyme du métabolisme des AG essentiels, et d'un désordre dans la composition des acides gras des phospholipides membranaires entraînant des modifications de la fluidité et de la perméabilité des membranes cellulaires. Chez le diabétique de type 2 , ces anomalies sont impliquées dans la physiopathologie des complications chroniques du diabète et en particulier les maladies cardiovasculaires et la neuropathie. Ce risque de complications pourrait être majoré chez le diabétique âgé. En effet, l'étude tunisienne (Sfar, 2010) réalisée auprès de 200 sujets en bonne santé, âgés de 40 à 82 ans, a montré que l'activité de la delta 6 désaturase diminue avec l'âge dans les deux sexes. Cette étude a également rapporté une association entre la composition des lipides plasmatiques en acides gras et les habitudes alimentaires en l'occurrence, une augmentation de la concentration en DHA et la consommation de poissons. Une autre étude tunisienne menée par KassabChekir (Kassab-Chekir, 2004) a révélé que la composition en acides gras polyinsaturés de la fraction des esters de cholestérol plasmatique notamment le pourcentage de l'acide alphalinolénique chez des diabétiques de type 2 ayant des complications cardiovasculaires $(0,63 \pm 0,15)$ est moins important que ceux des diabétiques non compliqués $(1,01 \pm 0,23)$ et des sujets témoins $(1,26 \pm 0,14)$. Donc, il serait intéressant d'étudier la composition des lipides plasmatiques en acides gras en fonction des habitudes alimentaires des diabétiques.

La limite majeure de notre étude était la petite taille de l'échantillon sélectionné qui n'est pas représentatif de la population diabétique de type 2 en Tunisie. Ce faible effectif est expliqué par la difficulté de la méthode utilisée pour l'évaluation des apports alimentaires (le semainier). En outre, la non disponibilité des teneurs en AGPI de certains aliments et la méconnaissance de l'influence des traitements thermiques et technologiques sur les teneurs en AGPI des aliments constituaient également des points faibles de l'étude.

\section{Conclusion}

Il ressort de notre étude, en dépit des imprécisions et des limites méthodologiques, un déséquilibre d'apport entre les 
deux précurseurs d'AGPI à longues chaînes, générant un rapport LA/ALA élevé, qui pourrait augmenter le risque des complications dégénératives notamment cardiovasculaires, chez nos diabétiques. Ces constats appellent à renforcer l'éducation nutritionnelle des diabétiques de type 2 et d'entreprendre les mesures nécessaires pour assurer un rapport LA/ALA optimal. Ces mesures consistent à faire un choix judicieux des huiles végétales afin de réduire la consommation de l'acide linoléique et d'augmenter celle de l'acide alphalinolénique. Certes, l'huile d'olive, fréquemment consommée en Tunisie, contribue via sa richesse en acide oléique à la prévention des maladies cardiovasculaires. Néanmoins, sa teneur faible en ALA incite à augmenter la consommation des huiles mélangées contenant notamment les huiles de soja et de colza. La couverture des besoins en ALA peut être également assurée par l'enrichissement de certains aliments couramment consommés. L'enrichissement peut être soit direct grâce à l'utilisation dans la formule des aliments d'ingrédients intrinsèquement riches en acides gras oméga 3 et autorisés en alimentation humaine, soit indirect via l'administration de graines de lin naturellement riches en acide alphalinolénique dans l'alimentation animale.

\section{Références}

Agence Française de Sécurité Sanitaire des Aliments (AFSSA). 2003. Rapport sur «Acides gras de la famille $\omega 3$ et système cardiovasculaire : intérêt nutritionnel et allégation », $54 \mathrm{p}$.

Agence nationale de sécurité sanitaire de l'alimentation, de l'environnement et du travail (ANSES). 2011. Actualisation des apports nutritionnels conseillés pour les acides gras. Rapport d'expertise collective. Disponible sur : http://www.anses.fr.

Astorg P. 2007. Apports en acides gras polyinsaturés notamment en DHA, dans la population française adulte : données issues de l'étude SU.VI.MAX et comparaisons avec d'autres études. $O C L$ 14: 28-34.

Centre d'information sur la qualité des aliments (CIQUAL). 2012. Disponible sur : www.afssa.fr/TableCIQUAL.

Dallongeville J. 2009. Acides gras $\omega 3$ et risque cardio-vasculaire. Méd. Maladies Métab. 3: 491-495.

Hulshof K., Van Erp-Baart MA, Anttolainen M, et al. 1999. Intake of fatty acids in Western Europe with emphasis on trans fatty acids : the TRANSFAIR study. Eur. J. Clin. Nutr. 53: 143-157.
Kassab-Chekir A, Ferchichi S, Kerkeni M, Hammemi S, Hammemi M, Laradi S, Miled A. 2004. Composition en acides gras des esters de cholestérol chez des diabétiques de type 2 tunisiens avec et sans complications cardiovasculaires. Ann. Biol. Clin. 62: 555562.

Kromhout D, Geleijnse JM, De Goede J, et al. 2011. N-3 Fatty Acids, Ventricular Arrhythmia-Related Events, and Fatal Myocardial Infarction in Postmyocardial Infarction Patients With Diabetes. Diabetes Care 34: 2515-2520.

Lecerf JM 2004. Poissons, acides gras $\omega 3$ et risque cardio-vasculaire. Cah. Nutr. Diét. 39: 143-150.

Lecerf JM. 2008. Acides gras et risque cardiovasculaire deuxième partie : Acides gras monoinsaturés et polyinsaturés oméga 6 . Cah. Nut. Diét. 44: 161-172.

Legrand P. 2007. Les acides gras : Structures, fonctions, apports nutritionnels conseillés. Cah. Nut. Diét. 42: 1S7-1S12.

Micha R, Khatibzadeh S, Shi P, et al. 2014. Global, regional, and national consumption levels of dietary fats and oils in 1990 and 2010: a systematic analysis including 266 country-specific nutrition surveys. BMJ 348: g2272.

Mnari Bhouri A, Jrah Harzallah H, Dhibi M, Bouhlel I, Hammami M, Chaouch A. 2009. Nutritional fatty acid quality of raw and cooked farmed and wild sea bream (Sparus aurata). J. Agricult. Food Chem. 58: 507-512.

Nettleton JA, Katz R. 2005. n-3 long-chain polyunsaturated fatty acids in type 2 diabetes: a review. J. Am. Diet. Assoc. 105: 350351.

Ollis TE, Meyer BJ, Howe PR. 1999. Australian food sources and intakes of omega-6 and omega-3 polyunsaturated fatty acids. Ann. Nutr. Metab. 43: 346-355.

Raccah D, Coste T, Gerbi A, Vague P. 1997. Les AGPI et le diabète. Cah. Nut. Diét. 32: 349.

Sfar S, Laporte F, Braham H, Jawed A, Amor S, Kerkeni A. 2010. Influence of dietary habits, age and gender on plasma fatty acids levels in a population of healthy Tunisian subjects. Exp. Gerontol. 45: 719-725.

Sugano M, Hirahara F. 2000. Polyunsaturated fatty acids in the food chain in Japan. Am. J. Clin. Nutr. 71: 189-196.

Tedgui A, chapman J. 2003. Pathogenèse de l'athérosclérose : théories et mécanismes. In : Toussant JF, Jacob MP, Lagrost L, Chapman J. L'athérosclérose : Physiologie, diagnostics, thérapeutiques. Paris : Masson, pp. 245-252.

Cite this article as: Henda Jamoussi, Salma Chaabouni, Amel Gammoudi, Faten Mahjoub, Kamilia Ounaissa, Olfa Berriche, Chiraz Amrouche, Samira Blouza. Apports spontanés en acides gras oméga 3 chez des diabétiques de type 2 tunisiens. OCL 2014, 21 (5) A501. 